\title{
地铁车辆轴箱吊耳断裂机理和试验研究"
}

\author{
石怀龙 王建斌 戴焕云 邬平波 \\ (西南交通大学牵引动力国家重点实验室 成都 610031)
}

\begin{abstract}
摘要: 地铁车辆在正常运营过程中发生轴箱吊耳断裂问题, 采用有限元分析方法和线路试验开展断裂机理研究, 并对吊耳振 动水平进行评估。通过分析振动激扰源和结构响应特性, 确定断裂原因和提出解决方案并进行试验验证。仿真表明吊耳第一 阶固有模态为横向弯曲, 主频约 $260 \mathrm{~Hz}$; 吊耳根部内圆弧处为强度薄弱点, 与现场裂纹位置吻合。试验表明轴箱体、吊耳振 动水平与线路区间相关, 钢轨波磨是导致车辆振动水平激增的主因, 波长 $61.5 \mathrm{~mm}$; 钢轨波磨波长、车辆常用速度共同作用 导致波磨频率在吊耳固有模态频带内, 导致结构共振从而引发疲劳破坏，提出钢轨打磨、优化吊耳结构设计和使用管理条件 等解决措施。开展钢轨打磨效果验证性试验, 表明钢轨打磨可显著降低吊耳加速度水平, 使结构应力降低 $50 \%$ 以上, 但部分 线路仍存在轻微波磨, 可根据车辆振动数据特征对波磨路段进行定位从而再次进行打磨。
\end{abstract}

关键词: 地铁车辆; 结构断裂; 钢轨波磨; 有限元分析; 线路试验

中图分类号: U270

\section{Crack Mechanism and Field Test of the Metro Safety Hanger}

\author{
SHI Huailong WANG Jianbin DAI Huanyun WU Pingbo
}

(State Key Laboratory of Traction Power, Southwest Jiaotong University, Chengdu 610031)

\begin{abstract}
There is crack found on the safety hanger mounted on the axle-box of railway metro. The finite element(FE) Analysis and field measurement were performed to study the crack mechanism, then the vibration level of the hanger is thoroughly evaluated. The excitation and natural response of the structure are analyzed, and solutions are provided and validated by the field measurement. The FE analysis shows that the lateral bending mode has the lowest frequency around $260 \mathrm{~Hz}$, and a weak area on the inner corner of the hanger bottom can be noticed. This shows a good agreement with the field crack propagation on the hanger. Field measurement illustrates that the vibration level of the axle-box and safety hanger are strongly dependent on the track segments. It is noticed that it is the rail corrugation with a wavelength of $61.5 \mathrm{~mm}$ that causes the serious vibration of vehicle. A resonance can be noticed by considering the natural mode of safety hanger and the track excitation frequency, which is dependent on the vehicle speed and corrugation wavelength. A fatigue problem can be encountered resulting from this resonance. Consequently, solutions for this structure failure issue are provided, such as the rail grinding, design a new safety hanger or specify the service conditions of this kind structure. Additionally, validation tests are conducted for the rail grinding solution which depicts that the rail grinding can significantly reduce the vibration level of vehicle, and the safety hanger stress reduces more than $50 \%$. However, there still exist corrugations on some track segments. The vehicle dynamic responses can be used to identify the corrugation level and location on track for further track maintenance.
\end{abstract}

Key words: railway metro; structure fracture; rail corrugation; finite element analysis; field measurement

\section{0 前言}

地铁车辆运营过程中具有空重车载重比大、汼 引/制动加速度大、线路曲线半径小等特点, 其运行 稳定性、安全性和平稳性指标与车辆运行状态直接 相关。地铁车辆在运行过程中会发生异常振动、噪

* 国家科技支撑计划资助项目(2015BAG13B01-03)。20180322 收到初稿, 20181210 收到修改稿
音、结构出现裂纹或者破坏等问题, 严重影响车辆 运行品质甚至是安全性。地铁车辆经常出现的问题 可以分为传动系统振动问题、构架主结构或者附属 部件强度问题、悬挂元件失效和车体振动或噪音问 题等。地铁车辆在牵引和制动过程中, 车体地板出 现显著弹性振动而引发脚麻问题, 试验研究发现是 车引传动系统中联轴节不对中效应所致 ${ }^{[1-2]}$ 。地铁车 辆转向架构架的齿轮箱吊挂座、横向止挡座等部位 出现裂纹, 可通过局部补强、提高焊缝强度等级等 
措施提升构架整体强度水平, 但需要同时采取降低 轨道不平顺水平和控制车速等措施 ${ }^{[3]}$ 。机车在低速 施加踏面制动时, 制动块出现颤振现象, 引发车辆 振动水平增大和产生噪音问题, 通过机理分析发现 制动块在低速制动情况下发生自激振动, 通过优化 转向架结构和悬挂参数可以得到改善 ${ }^{[4]}$ 。采用橡胶 轮的地铁车辆由于轮胎偏磨而导致车体抖动, 需要 对车轮磨耗状态进行管理 ${ }^{[5]}$ 。由于钢轨的短波波磨 $(30 \sim 40 \mathrm{~mm})$ 造成车辆振动水平恶化而引发一系钢 弹簧和安全吊断裂, 属于振动疲劳问题, 需要通过 控制车速和钢轨打磨解决 ${ }^{[6-8]}$ 。北京地铁波磨试验研 究发现, 剪切型钢轨扣件会导致轮轨接触产生 200 400 Hz 左右的共振, 造成使用该型号扣件路 段产生波长为 55 63 mm 的波磨, 通过增加橡胶垫 块可缓解波磨问题 ${ }^{[9-10]}$ 。不同类型的钢轨扣件由于 刚度分配不同, 从而造成不同波长的波磨, 并与轨 道结构的垂弯振动特性密切相关 ${ }^{[11]}$ 。钢轨波磨特 征、产生机理和打磨措施等与轨道结构、扣件参数、 车辆运行状态和线路维护情况等相关 ${ }^{[12-13]}$ 。车轮缺 陷导致的轮对冲击载荷, 会对轮轴、构架结构疲劳 产生显著影响 ${ }^{[14-15]}$, 而基于断裂力学的结构疲劳裂 纹萌生与扩展研究可从机理上解释结构部件的疲劳 断裂问题 ${ }^{[16]}$ 。车辆结构部件的强度和异常振动问 题, 可以从振动激扰源、振动传递路径和结构振动 响应三方面进行分析, 并且结构破坏属于振动疲劳 问题。上述地铁车辆遇到的问题均与车辆运行速度、 车轮/钢轨磨耗状态、车辆悬挂参数相关, 需要综合 考虑这些参数才能确定问题来源并提出对症的解决 方案。

本文针对地铁车辆出现的轴箱吊耳断裂㕫题开 展机理分析和试验研究, 采用有限元分析和线路测 试相结合的方法, 分析振动激扰源和结构固有响应 特性, 确定轴箱吊耳断裂原因和提出解决方案并进 行试验验证。首先, 描述轴箱吊耳断裂问题, 并开 展结构模态分析和强度校核计算, 指出结构振动形 式和强度薄弱位置。然后, 制定试验方案并开展线 路振动环境测试, 分别从时域幅值对比和频域特征 分析轴箱体、轴箱吊耳的振动水平和应力等级, 确 定轴箱吊耳应力水平恶化原因。再有, 提出 3 种可 能的解决方案, 基于车辆振动数据特征识别钢轨波 磨程度并对波磨路段进行定位, 对钢轨打磨方案进 行试验验证。

\section{1 轴箱吊耳断裂问题描述}

地铁车辆在正常运营过程中发生轴箱吊耳断裂
问题, 如图 1 所示。吊耳通过螺栓刚性连接在轴箱 体端部, 属于轴箱体附件。车辆在正常运行时轴箱 吊耳不发挥任何功能, 仅在对整车进行起吊作业时 同构架限位止挡配合，发挥一系垂向相对位移限位 的作用。图 1 显示吊耳根部出现横裂纹, 裂纹起源 于内圆弧处, 然后再逐渐向外圆弧处扩展。统计其 它轴箱吊耳断裂脱落案例, 发现裂纹源均在根部的 内圆弧处, 即该处为结构应力薄弱位置, 而螺栓连 接处则未曾出现该问题。由于车辆在正常运行时轴 箱吊耳和限位止挡不会出现接触或碰撞, 故应该从 轴箱体激扰水平和结构本身查找原因。

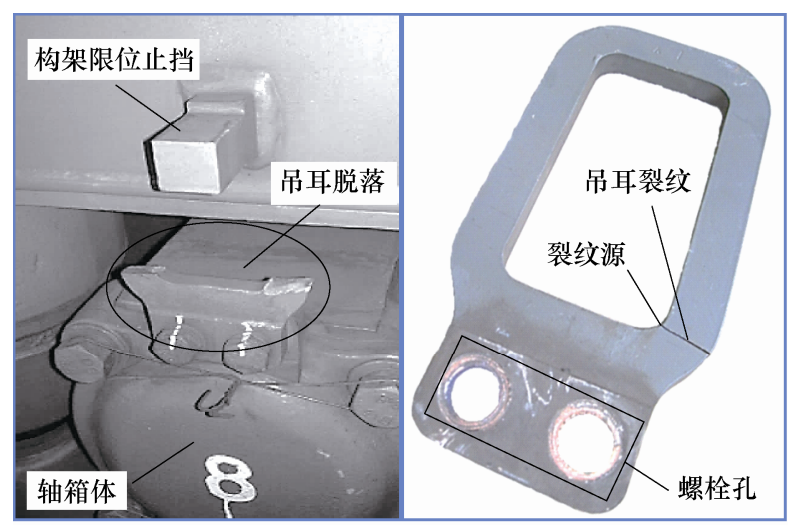

图 1 轴箱吊耳断裂实物图

\section{2 结构模态与强度校核计算}

为确定轴箱吊耳在现有安装方式下的结构固有 振动特性, 需要进行结构模态和强度校核计算, 在 螺栓安装面施加位移约束以模拟吊耳与轴箱体刚性 连接的边界条件。吊耳总长度和宽度分别为 205 $\mathrm{mm} 、 105 \mathrm{~mm}$, 根部厚度 $16 \mathrm{~mm}$, 主结构厚度 $12 \mathrm{~mm}$ 。 采用六面实体单元进行自由网格划分, 节点数为 34025 , 单元数为 7463 , 单元最大长度 $3 \mathrm{~mm}$ 。结 构材料为 $\mathrm{Q} 235 \mathrm{~B}$, 屈服强度为 $225 \mathrm{MPa}^{[17]}$, 度劳许 用应力为 $110 \mathrm{MPa}$ 。

模态计算结构如图 2a 2c 所示, 分别对应为横 向弯曲模态 $260.4 \mathrm{~Hz}$ 、纵向弯曲模态 $793.3 \mathrm{~Hz}$ 和扭 转模态 $798.8 \mathrm{~Hz}$ 。从振型图可以看出, 轴箱吊耳主 要振型为上部发生横向、纵向和扭转位移, 而根部 变形小为主要应力集中区。最低阶模态为横向弯曲 模态, 为轴箱吊耳的主要结构振动形式。

参考 IEC61373-1999 标准按轴端部件施加冲 击载荷和常规载荷, 对轴箱吊耳进行冲击试验强 度校核和长寿命试验强度校核。三向冲击试验载 荷幅值均为 $1000 \mathrm{~m} / \mathrm{s}^{2}$, 长寿命试验加速度为垂向 $300 \mathrm{~m} / \mathrm{s}^{2}$ 、横向 $270 \mathrm{~m} / \mathrm{s}^{2}$, 纵向 $135 \mathrm{~m} / \mathrm{s}^{2[18]}$ 。由于 地铁项目完成时, 新版标准 EN61373-2010 还未生 
效, 本文仍采用旧版数值执行长寿命疲劳强度校 核。图 $2 \mathrm{~d}$ 为强度校核结果, 标记出了最大等效应 力出现位置。两种计算工况下的最大等效应力均 出现在根部内圆弧处, 等效应力分别为 $103.1 \mathrm{MPa}$ 和 $24.8 \mathrm{MPa}$, 远小于材料的屈服强度 $225 \mathrm{MPa}$ 和 疲劳强度 $110 \mathrm{MPa}$, 表明结构强度设计已经富有足 够的安全裕量。

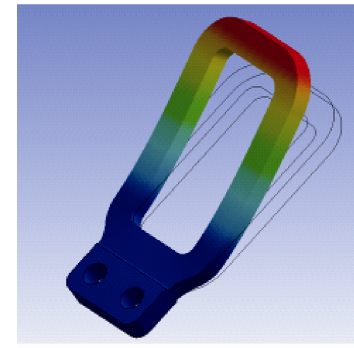

(a) 横向弯曲 $260.4 \mathrm{~Hz}$

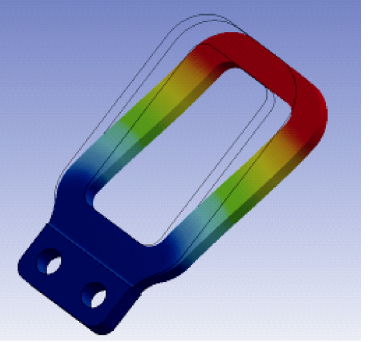

(b) 纵向弯曲 $793.3 \mathrm{~Hz}$

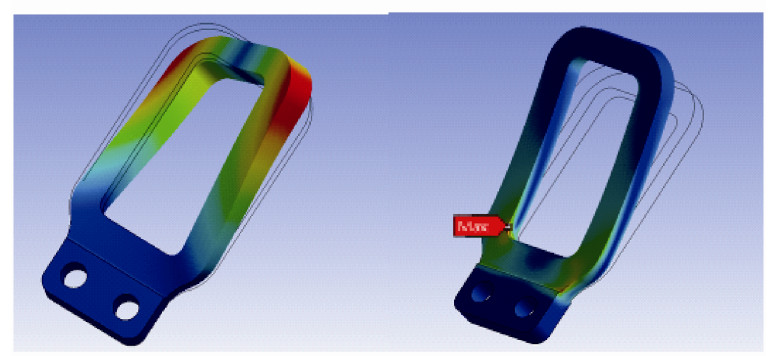

(c) 扭转模态 $798.8 \mathrm{~Hz}$

(d) 等效应力最大点
图 2 轴箱吊耳模态振型和等效应力云图

对于结构简单部件, 可以通过仿真计算建立 载荷和应变的关系, 便于后文根据振动加速度直 接估算其结构应力大小, 即建立载荷-应力关系。 由于轴箱吊耳以横向弯曲振动为主, 且强度薄弱 点为根部内圆弧处, 可建立横向加速度-内圆弧处 应力之间的对应关系。根据疲劳许用应力极限允许 值反推载荷, 对轴箱吊耳在承受最大应力 $110 \mathrm{MPa}$ 时所施加的横向加速度进行预测, 结果为 $1420 \mathrm{~m} / \mathrm{s}^{2}$ (约 145g)。

\section{3 线路振动环境测试}

\section{1 试验方案}

由于吊耳直接安装在轴箱体上, 因此测试了轴 箱体和吊耳加速度, 并采集吊耳断裂处的动应力水 平, 如图 3 所示。轴箱加速度计位于箱体顶部, 吊 耳加速度计位于结构顶部, 并在吊耳根部布置了 4 个单向应变计。采用压电式高频响加速度计, 量程 $500 \mathrm{~g}$, 分辨率 $0.002 \mathrm{~g}$, 抗冲击载荷 $1000 \mathrm{~g}$, 有效带 宽 $1 \sim 12 \mathrm{kHz}$ 。电阻应变计宽度 $6 \mathrm{~mm}, 120 \Omega$, 最 高频响 $5 \mathrm{kHz}, 1 / 4$ 惠斯通桥路组桥。数据采集系统 还包括直流供电模块、数采系统和控制计算机, 试
验中设定最高数据采样频率 $5 \mathrm{kHz}$ 。在运营线路上 进行试验, 车辆为空车即不载客状态, 列车采用自 动驾驶模式 ATO 速度运行, 最高车速 $72 \mathrm{~km} / \mathrm{h}$, 常 用速度 $40 \sim 70 \mathrm{~km} / \mathrm{h}$ 。线路已营运多年, 全长 $23 \mathrm{~km}$, 正线曲线半径 380 1 $200 \mathrm{~m}$; 共计 13 座车站, 单程 运行 33 min。车辆采用 6 辆编组 3 动 3 拖配置方式, 其中 $\mathrm{Mc} 、 \mathrm{Mc} 4$ 和 $\mathrm{Mc} 5$ 为动车, $\mathrm{Tc} 1 、 \mathrm{Tc} 3$ 和 $\mathrm{Tc} 6$ 为拖车, 被试车辆为动车 $\mathrm{Mc} 2$ 二位端转向架。

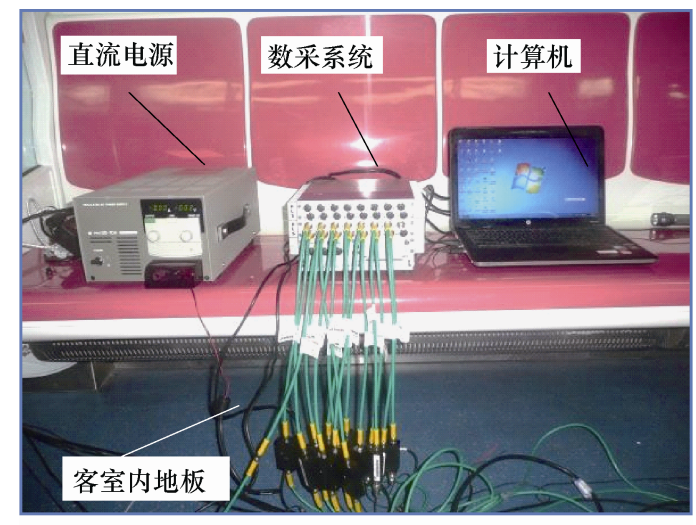

(a) 数据采集系统

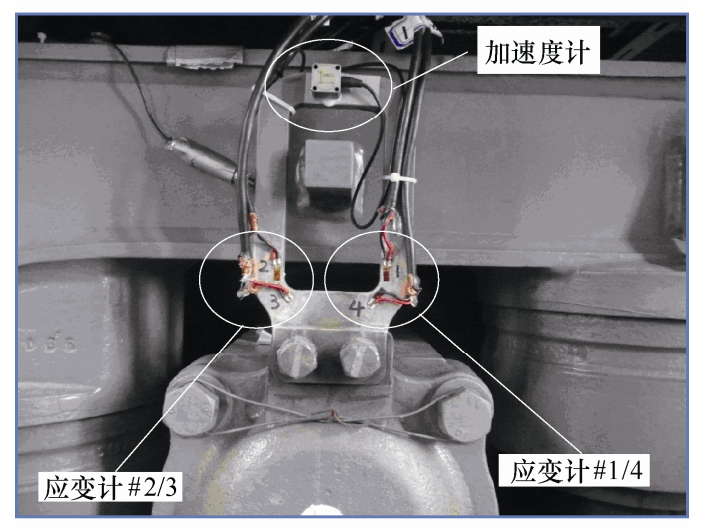

(b) 传感器布置

图 3 轴箱吊耳振动水平测试现场图

\section{2 时域振动水平对比}

统计所有站间的轴箱体、吊耳加速度和应力水 平，对比不同线路区间内的振动响应幅值，如表 1 、 2 所示。将原始时程曲线每隔 $6 \mathrm{~s}$ 分段, 找出各段的 幅值最大值, 然后再平均这些最大值作为该线路区 间的幅值平均最大值, 该指标可有效表达车辆在该 线路区间内的整体振动水平。

由表 1 可知，区间 $S 9 \sim \mathrm{S} 13$ 的轴箱体和吊耳振 动水平显著高于区间 $\mathrm{S} 1 \sim \mathrm{S} 8$, 加速度幅值放大至 $2 \sim 6$ 倍, 而吊耳动力应力水平也显著增加, 应力幅 值放大至 1.5 2 倍, 幅值平均值相差也较大。对比 横向、垂向振动水平发现, 轴箱体垂向加速度平均 值约为横向的 2.5 倍, 而吊耳横向加速度平均值约 为垂向的 2 倍。因此, 基于上述数据分析可以判定 
区间 S9 S13 可能存在异常的轨道激扰, 如钢轨波 磨, 造成轴箱体垂向加速度显著大于横向。吊耳动 应力幅值平均最大值表明, 区间 S5、S9 S13 的结 构应力为 $85.1 \sim 177.1 \mathrm{MPa}$, 其应力最大值则显著大 于 $110 \mathrm{MPa}$, 即大于材料的疲劳寿命许用极限, 结 合后文关于载荷作用频次统计结果, 认为这些路段 的异常轨道激扰会显著降低吊耳的使用寿命。

\section{表 1 轴箱体、吊耳加速度和应力平均最大值统计表}

\begin{tabular}{|c|c|c|c|c|c|}
\hline 线路 & $\begin{array}{l}\text { 轴箱横向 } \\
\text { 加速度 } / g\end{array}$ & $\begin{array}{l}\text { 轴箱垂向 } \\
\text { 加速度 } / g\end{array}$ & $\begin{array}{l}\text { 吊耳横向 } \\
\text { 加速度 } / g\end{array}$ & $\begin{array}{l}\text { 吊耳垂向 } \\
\text { 加速度/g }\end{array}$ & $\begin{array}{c}\text { 吊耳应力 } \\
/ \mathrm{MPa}\end{array}$ \\
\hline S1 & 6.9 & 26.9 & 32.1 & 21.5 & 48.1 \\
\hline S2 & 6.1 & 19.1 & 27.5 & 16.5 & 52.7 \\
\hline S3 & 6.3 & 17.3 & 28.4 & 14.9 & 59.6 \\
\hline S4 & 6.0 & 15.2 & 30.4 & 13.6 & 73.9 \\
\hline$\underline{\mathrm{S} 5}$ & $\underline{6.4}$ & $\underline{14.9}$ & $\underline{40.7}$ & $\underline{15.3}$ & $\underline{85.1}$ \\
\hline S6 & 5.0 & 10.1 & 26.7 & 10.4 & 56.4 \\
\hline S7 & 8.8 & 23.9 & 35.8 & 11.9 & 60.6 \\
\hline S8 & 5.0 & 12.0 & 20.4 & 9.4 & 43.9 \\
\hline$\underline{\mathrm{S} 9}$ & $\underline{10.1}$ & $\underline{21.2}$ & $\underline{42.0}$ & $\underline{13.0}$ & $\underline{109.8}$ \\
\hline$\underline{\mathrm{S} 10}$ & $\underline{38.4}$ & $\underline{92.2}$ & $\underline{61.3}$ & $\underline{19.4}$ & $\underline{116.9}$ \\
\hline$\underline{\mathrm{S} 11}$ & $\underline{18.3}$ & $\underline{47.6}$ & $\underline{42.3}$ & $\underline{15.3}$ & $\underline{90.2}$ \\
\hline$\underline{\mathrm{S} 12}$ & $\underline{10.1}$ & $\underline{20.1}$ & 45.7 & $\underline{18.1}$ & $\underline{93.3}$ \\
\hline$\underline{\mathrm{S} 13}$ & $\underline{17.0}$ & $\underline{46.0}$ & $\underline{78.0}$ & $\underline{37.6}$ & $\underline{177.1}$ \\
\hline $\begin{array}{c}\text { S9 S13 } \\
\text { 均值 }\end{array}$ & 18.8 & 45.4 & 53.9 & 20.7 & 117.5 \\
\hline $\begin{array}{c}\mathrm{S} 1 \sim \mathrm{S} 8 \\
\text { 均值 }\end{array}$ & 6.3 & 17.4 & 30.4 & 12.6 & 64.9 \\
\hline
\end{tabular}

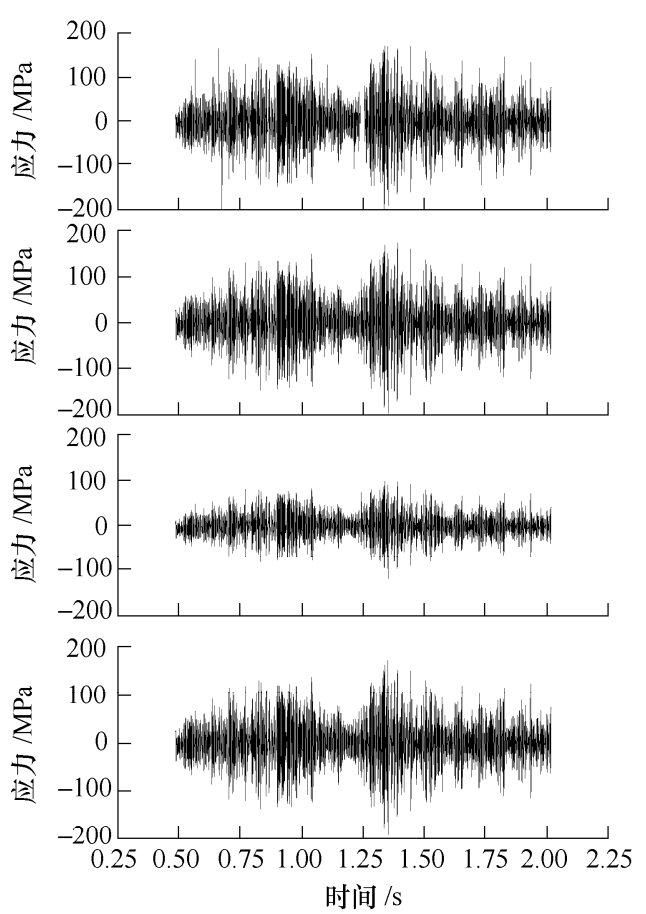

(a)

\section{3 频域振动特征分析}

图 4 为 $\mathrm{S} 13$ 线路区间内车速 $70 \mathrm{~km} / \mathrm{h}$ 时吊耳的 横向加速度时程曲线和频谱图。时程曲线表明, 横向加速度幅值最大值接近传感器量程约 $500 \mathrm{~g}$, 并且幅值超过疲劳许用加速度 $145 \mathrm{~g}$ 次数非常多, 表明吊耳振动水平相当恶劣。频谱图可以解释吊 耳振动水平过大的原因, 振动主频分别为 $260 \mathrm{~Hz}$ 和 $318 \mathrm{~Hz}$, 前者带宽较宽对应吊耳的横向弯曲模 态, 后者频带窄对应钢轨波磨频率。图 5 为 4 个 测点处的动应力时程曲线和频谱图, 吊耳单向应 力幅值显著大于 $110 \mathrm{MPa}$, 并且频次非常多, 表明 吊耳处于高应力水平; 频谱分析显示钢轨波磨频 率在结构固有模态频带内, 是造成吊耳应力水平 显著增加的主因, 即是钢轨波磨导致的结构共振 从而引发疲劳破坏。

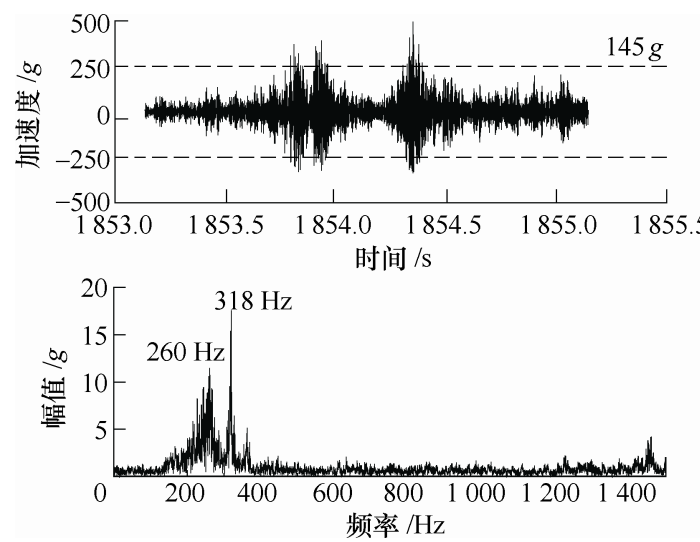

图 4 轴箱吊耳横向加速度时域和频谱图

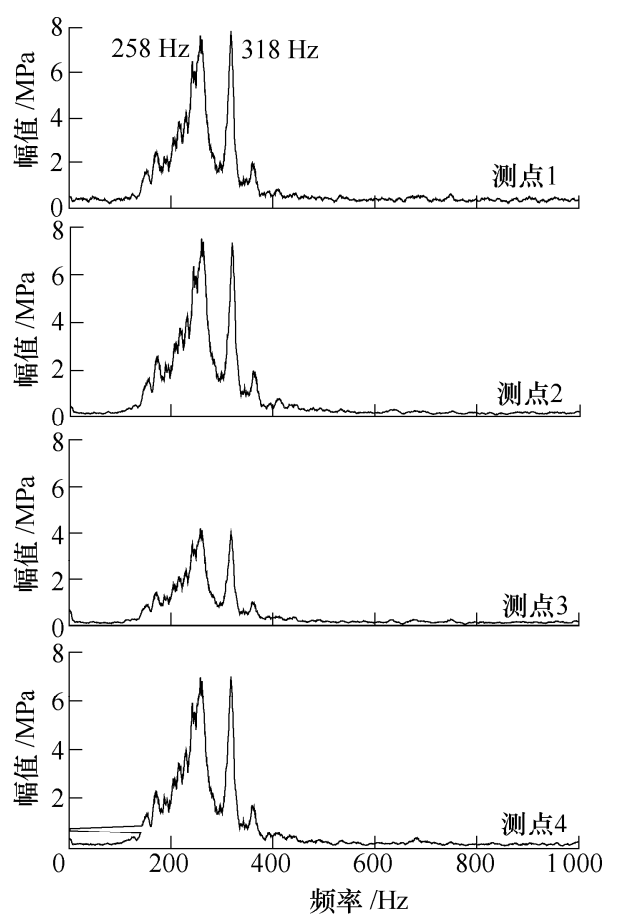

(b)

图 5 轴箱吊耳动应力时程曲线和频谱图 
已知当前车速为 $v=70 \mathrm{~km} / \mathrm{h}$, 则可计算出钢轨波 磨波长为 $l=v / f=61.5 \mathrm{~mm}$, 已知 $f=318 \mathrm{~Hz}$ 。根据车速、 线路平面图可以计算出具体公里标, 结合车辆系统 的振动响应特征, 可识别出振动水平偏大路段的地 理位置, 从而可以上线勘察线路、钢轨状态。实地 观察发现, 轴箱体振动水平显著增加路段的钢轨存 在明显的波磨, 波长与试验数据推算结果基本一致, 从而判定钢轨波磨是导致车辆振动水平激增的主 因, 并且为吊耳断裂的振动激扰源。通过对全程振 动数据进行时域、频域分析, 可识别出不同路段的 波磨程度和详细的公里标, 为线路维护部门进行线 路整治提供参考。

\section{4 解决方案}

综合分析试验数据结果, 能够解决轴箱吊耳断 裂问题的主要方案如下。

(1) 通过钢轨打磨降低轨道激扰水平。根据实 测的轴箱体加速度信号和车速信息等, 可给出以线 路公里标形式的波磨路段具体位置, 然后进行钢轨 打磨。受限于钢轨打磨程度、技术水平等因素影响, 实际上并不能完全消除钢轨波磨。因此, 打磨后的 钢轨在一定程度上仍有轻微的波磨, 仍会对车辆系 统施加高频激扰。

(2) 轴箱吊耳使用条件管理。考虑到轴箱吊耳 仅在整车起吊时起到限位作用, 而当车辆正常运行 时不发挥任何作用, 因此, 可考虑拆除且仅在整车 起吊时才安装。

（3）新型轴箱吊耳结构设计。由于常用车速比 较固定, 则需要将结构固有模态频率避开波磨激扰 频段。由于钢轨波磨激扰频带较宽, 这给新型结 构模态设计带来难度, 类似结构也会出现同样的问 题 $^{[7]}$ 。因此, 可考虑改变吊耳安装位置, 如安装在 构架上, 限位止挡放在轴箱体上, 因为构架振动 水平比轴箱体小一个数量级, 从而改善了吊耳振 动环境。

\section{5 钢轨打磨效果验证试验}

通过对钢轨进行打磨以降低波磨程度, 然后 再次进行验证试验。钢轨打磨前后的吊耳横向、 垂向加速度幅值统计如图 6 所示, 采用了穿级计 数统计方法 ${ }^{[19]}$ 。钢轨打磨可显著降低吊耳的加速度 水平。以横向加速度为例, 打磨前后对应幅值大于 $145 \mathrm{~g}$ 共计 2295 次和 180 次, 垂向振动也具有类似 的规律。

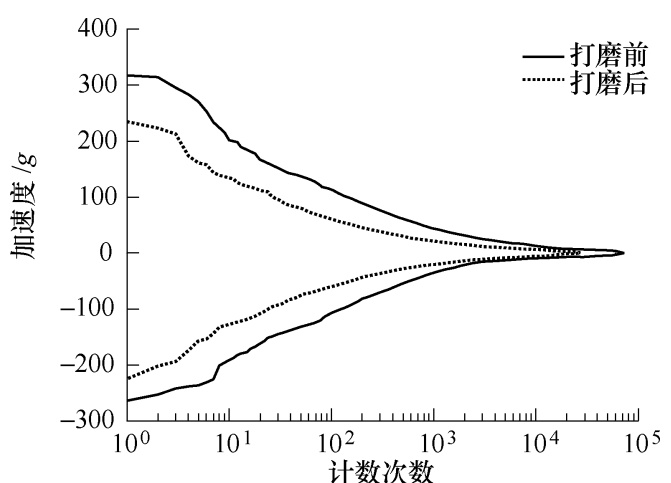

(a) 垂向

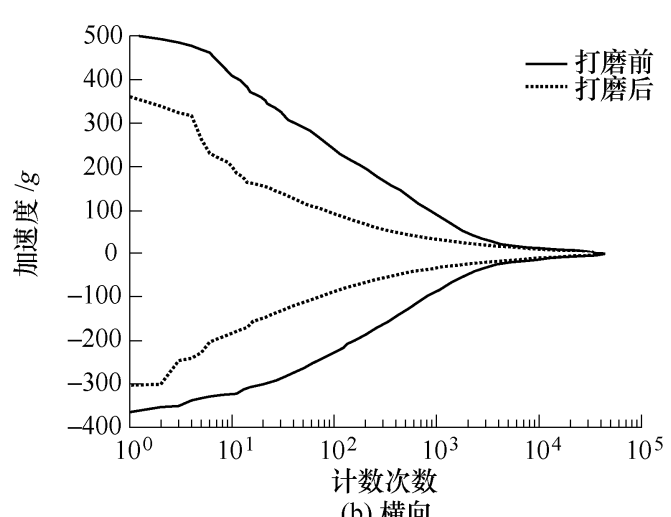

图 6 轴箱吊耳振动加速度幅值穿级计数统计

吊耳测点 1 处动应力方均根值对比如图 7 所示, 对整条线路钢轨进行打磨可使结构应力水平降低 $50 \%$ 以上。图 8 为吊耳横向加速度时程曲线和频谱, 这里仅给出整条线路上振动水平仍相对较大区段的 测试结果。频谱分析表明，钢轨打磨后吊耳固有模 态振动 $(260 \mathrm{~Hz})$ 不显著, 振动主频单一且频率约为 $331 \mathrm{~Hz}$ ，即仍存在钢轨打磨前的波磨频率 $318 \mathrm{~Hz}$, 并且成为吊耳的主要振动形式。时程曲线表明, 钢 轨打磨可显著降低幅值, 振动最大路段的加速度幅 值远小于 $145 \mathrm{~g}$, 即能够满足材料疲劳载荷许用极限 要求。综上所述，钢轨打磨前后的吊耳横向、垂向 加速度和动应力数据表明, 钢轨打磨使吊耳振动水 平显著降低，最大载荷低于疲劳许用极限载荷，有

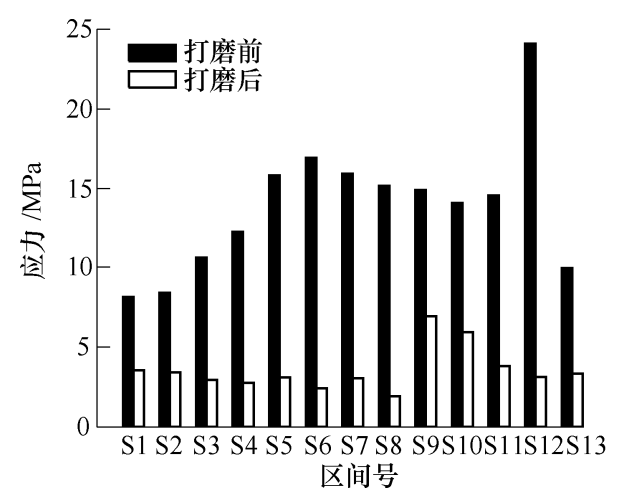

图 7 轴箱吊耳动应力方均根值对比直方图 
助于提高其使用寿命; 但部分线路区间仍存在一定 程度的波磨, 不同路段的吊耳振动水平有差异, 在 部分区间振动加速度仍较大, 可通过车辆振动数据 识别出具体的线路位置并再次进行打磨。

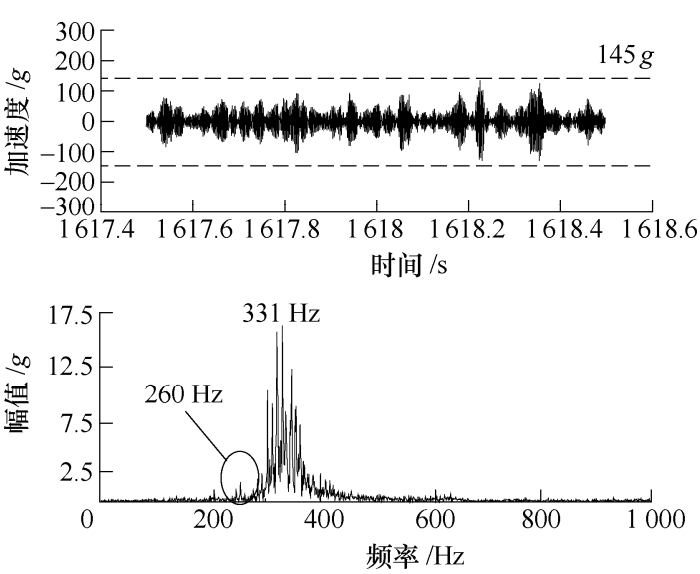

图 8 轴箱吊耳横向加速度时域和频谱图

\section{6 结论}

本文针对地铁车辆轴箱吊耳断裂问题开展机理 分析和试验研究, 采用有限元仿真和线路测试方法 查找振动激扰源和结构响应特性, 分析吊耳断裂机 理并提出解决方案和进行试验验证。

(1) 有限元分析表明, 吊耳第一阶固有模态为 横向弯曲模态, 频率为 $260 \mathrm{~Hz}$; 强度计算结果显示 应力集中点为吊耳根部内圆弧处, 与现场裂纹位置 吻合; 根据疲劳极限(110 MPa)计算出对应的横向许 用载荷为 $145 g$ 。

(2) 试验表明轴箱体、吊耳振动水平与线路区 间相关。部分路段内振动水平显著增大, 轴箱体、 吊耳的加速度幅值平均最大值放大至 2 6 倍, 动应 力幅值平均最大值放大至 $1.5 \sim 2$ 倍, 超出吊耳疲劳 强度许用极限。轴箱体垂向加速度平均值约为横向 的 2.5 倍, 主要受钢轨波磨影响; 吊耳横向加速度 平均值约为垂向的 2 倍, 主要受横向弯曲固有模态 振动影响。

(3) 试验表明车辆常用速度导致钢轨波磨频率 在吊耳固有模态频带内, 从而引发结构共振导致其 应力水平激增, 车速 $40 \sim 70 \mathrm{~km} / \mathrm{h}$, 波长 $61.5 \mathrm{~mm}$ 。

(4) 钢轨打磨可显著降低吊耳的加速度水平, 并使应力水平降低 $50 \%$ 以上, 可显著提高使用寿命; 部分线路仍存在一定程度的波磨, 可根据车辆振动 数据识别出波磨路段位置从而再次进行打磨。

\section{参 考 文 献}

[1] 宋庆伟, 李国栋, 高纯友, 等. 北京地铁亦庄线车辆异
常振动研究 $[J]$. 铁道车辆, 2014, 52(5): 5-8.

SONG Qingwei, LI Guodong, GAO Chunyou, et al. Research on the abnormal vibration of vehicles on Yizhuang line of Beijing metro[J]. Rolling Stock，2014, 52(5): 5-8.

[2] 石怀龙, 王建斌, 戴焕云, 等. 联轴器不对中导致的车 体振动研究 [J]. 振动、测试与诊断, 2015，35(4): 626-631.

SHI Huailong, WANG Jianbin, DAI Huanyun, et at. Car body vibration caused by the misalignment of rotor-shaft coupling of Metro[J]. Journal of Vibration, Measurement \& Diagnosis, 2015, 35(4): 626-631.

[3] 耿幸福, 员华. 地铁转向架构架疲劳开裂分析与改造方 案 $[\mathrm{J}$. 都市快轨交通，2010，23(6): 90-93.

GENG Xingfu, YUAN Hua. Fatigue crack analysis and restoration options for metro vehicle bogie frames[J]. Urban Rapid Rail Transit， 2010， 23(6): 90-93.

[4] 戴焕云. 制动工况下机车车辆转向架颤振机理 [J]. 交 通运输工程学报，2005，5(3)：5-7.

DAI Huanyun. Chatter mechanism of railway vehicle bogie under braking condition[J]. Journal of Traffic and Transportation Engineering, 2005，5(3): 5-7.

[5] 王军. 广州地铁 APM 线列车异常轻微抖动故障的分 析与处理[J]. 轨道交通装备与技术, 2015, 34(4): 34-36. WANG Jun. Analysis and solution for the abnormal vibration of Guangzhou Metro APM[J]. Rail Transportation Equipment and Technology, 2015, 34(4): 34-36.

[6] 袁浩智. 广州地铁三号线北延段列车一系钢弹簧断裂 原因分析及处理 [J]. 轨道交通装备与技术, 2015, 1(1): 32-34.

YUAN Haozhi. Analysis and solution for the primary steel spring crack of Guangzhou Metro Line 3[J]. Rail Transportation Equipment and Technology，2015， 1(1): 32-34.

[7] 张春玉, 程亚军, 邵俊捷. 基于线路实测载荷谱的转向 架部件振动疲劳分析 [J]. 城市轨道交通研究, 2017, 20(2): 46-47.

ZHANG Chunyu, CHENG Yajun, SHAO Junjie. Vibration fatigue analysis of bogie components based on routine test load spectrum[J]. Urban Mass Transit, 2017, 20(2): 46-47.

[8] 李伟, 曾全君, 朱士友, 等. 地铁钢轨波磨对车辆和轨 道动态行为的影响 [J]. 交通运输工程学报, 2015, 15(1): 34-42.

LI Wei, ZENG Quanjun, ZHU Shiyou, et al. Effect of metro rail corrugation on dynamic behaviors of vehicle and track[J]. Journal of Traffic and Transportation Engineering, 2015, 15(1): 34-42.

[9] 刘维宁, 任静, 刘卫丰, 等. 北京地铁钢轨波磨测试分 
析[J]. 都市快轨交通, 2011，24(3): 6-9.

LIU Weining, REN Jing, LIU Weifeng, et al. Tests and analysis on rail corrugation of Beijing Metro[J]. Urban Rapid Rail Transit, 2011，24(3): 6-9.

[10] 刘卫丰, 刘维宁, 吴宗臻, 等. 北京地铁剪切型减振器 扣件钢轨波磨治理的试验研究 [J]. 机械工程学报, 2015, 51(21): 26-32.

LIU Weifeng, LIU Weining, WU Zongzhen, et al. Test study on treating rail corrugation for egg fastener in Beijing metro[J]. Journal of Mechanical Engineering, 2015, 51(21): 26-32.

[11] EGANA J, VINOLAS J, SECO M. Investigation of the influence of rail pad stiffness on rail corrugation on a transit system[J]. Wear, 2006, 261(2): 216-224.

[12] GRASSIE S L. Rail corrugation: Characteristics, causes and treatments $[\mathrm{J}]$. Proceedings of the Institution of Mechanical Engineers, Part F: Journal of Rail and Rapid Transit, 2009, 223(6): 581-596.

[13] JIN Xuesong, WEN Zefeng, WANG Kaiyun. Effect of track irregularities on initiation and evolution of rail corrugation[J]. Journal of Sound and Vibration, 2005, 285(1-2): 121-148.

[14] 王建斌, 邬平波, 唐兆. 车轮扁疮引发附加冲击力对车 轴应力谱影响的研究 [J]. 铁道学报, 2006, 28(1): 39-43. WANG Jianbin, WU Pingbo, TANG Zhao. Research on the axle stress spectrum considering extra wheel flat impact forces[J]. Journal of the China Railway Society, 2006, 28(1): 39-43.

[15] 肖守讷, 李华丽, 阳光武, 等. 轮轨冲击对构架疲劳的
影响 $[\mathrm{J}]$. 交通运输工程学报, 2008, 8(3): 6-9.

XIAO Shoune, LI Huali, YANG Guangwu, et al. Influence of wheel-rail impact on fatigue of bogie frame[J]. Journal of Traffic and Transportation Engineering, 2008, 8(3): 6-9.

[16] 吴圣川, 吴玉程. 断裂分析及 CAE 软件的现状与发展 [J]. 计算机辅助工程, 2011，20(1): 1-2.

WU Shengchuan, WU Yucheng. Review of the crack analysis and CAE software development[J]. Computer Aided Engineering, 2011，20(1): 1-2.

[17] 中华人民共和国国家质量监督检验检疫总局, 中国国 家标准化管理委员会. GB/T 700-2006 碳素结构钢[S]. 北京: 中国标准出版社, 2006.

General Administration of Quality Supervision, Inspection and Quarantine of the People's Republic of China, Standardization Administration of the People's Republic of China. GB/T 700-2006 Carbon structural steels[S]. Beijing: Standards Press of China, 2006.

[18] IEC61373. Railway applications-Rolling stock equipmentshock and vibration tests[S]. International Electrotechnical Commission, 1999.

[19] TOVO R. Cycle distribution and fatigue damage under broad-band random loading[J]. International Journal of Fatigue, 2002, 24(11): 1137-1147.

作者简介: 石怀龙, 男, 1986 年出生, 博士, 助理研究员。主要研究方 向为机车车辆系统动力学。

E-mail: shi@swjtu.cn

邬平波(通信作者), 男, 1968 年出生, 博士, 研究员, 博士研究生导师。 主要研究方向为机车车辆系统动力学与强度。

E-mail: wupingbo@163.com 